\title{
Televisión y diversidad: \\ Génesis, definiciones y perspectivas de la diversidad en las televisiones públicas y comerciales
}

\author{
Charo Gutiérrez Gea \\ Investigadora LAPREC \\ (Laboratorio de Prospectiva en Investigación en Comunicación)
}

\begin{abstract}
Este artículo presenta un análisis de la génesis del valor de la diversidad dentro de los medios de comunicación, y especialmente en las televisiones públicas y comerciales, de los elementos más significativos que se encuentran planteados en el debate sobre la diversidad en algunos países desarrollados, y algunas de las definiciones que se han elaborado sobre este concepto. También, en este artículo se analiza las principales perspectivas de investigación sobre las dimensiones de la diversidad y la importancia que tiene este valor en la actual era multicanal, en relación, especialmente con el futuro de las televisiones públicas.
\end{abstract}

\section{Introducción}

$\mathbf{E}$

1 concepto de la diversidad está vinculado a dos debates intelectuales, por una parte está vinculado con los rasgos claves de la comunicación de masas, y por otra, con la reflexión que se hace desde la Ciencia Política sobre el pluralismo y el sistema democrático. En los dos debates, la diversidad está considerada como una forma de valor social positivo. Dentro de los medios de comunicación el valor de la diversidad puede verse limitado por factores políticos, económicos y sociales. Dependiendo de análisis ideológicos divergentes, este valor puede disminuir, tanto por la intervención del Estado que tiende a regular y controlar los medios de comunicación; como por la concentración de la propiedad dentro del sistema de medios; o las tendencias internas de las instituciones mediáticas. La diversidad dentro de los medios de comunicación, y especialmente de la televisión, se intenta desarrollar, a través de políticas comunicativas contradictorias: la primera es mediante la ampliación de los canales existentes; y la segunda, es a través de la intervención pública y la regulación del mercado.

Históricamente, las televisiones públicas han estado orientadas hacia el desarrollo del pluralismo programático, tanto regional, lingüístico, político, cultural y en materia de gustos, unido el acceso de la sociedad a una información pluralista. Actualmente, el valor de diversidad es un principio básico dentro de la filosofía del servicio público, donde las instituciones públicas de televisión deben servir al público, reconocer los derechos de los ciudadanos en la sociedad y actuar 
como la mejor fuente posible de información para apoyar el diálogo público. En las reflexiones actuales que se están realizando sobre las televisiones públicas, en los países desarrollados, uno de los conceptos básicos asociados al servicio público ha sido la diversidad, que debe desarrollarse dentro del medio público, para favorece runa información y opiniones plurales.

En las distintas reflexiones e investigaciones que se han realizado sobre el valor de la diversidad, se considera que es posible estudiar y medir con precisión las diferentes dimensiones asociadas con esta valor. Estas dimensiones pueden ser: la diversificación de la programación de un sistema o canal de televisión; la diversidad en la representación social; la diversidad estilística, de la audiencia, de contenidos, geográfica, de formatos y metas y de opiniones, y pueden medirse con cierta precisión, a través de una serie de indicadores empíricos.

\section{Génesis del concepto de diversidad}

El valor de la diversidad ha nacido principalmente a partir de dos debates intelectuales: el primero está relacionado con la sociedad de masas; y el segundo, con el ámbito de la teoría política, vinculado con el tema del Estado democrático liberal.

En relación al debate sobre la sociedad de masas, un elemento importante de la definición de la sociedad de masas, ha sido la uniformidad cultural y la concentración de poder en manos de una autoridad socialmente alejada. La sociedad de masas se caracteriza por tener una gran homogeneidad cultural, por un alto grado de individualismo y por tener unos vínculos sociales débiles. También, la sociedad de masas se caracteriza por una relativa ausencia de asociaciones intermediarias entre el individuo y el Estado (Mcquail y Cuilenburg, 1983: 146 y Ss).

En la sociedad de masas, los medios de comunicación han favorecido y reforzado los rasgos que tienden a la estandarización masificada, dirigiendo su atención hacia los centros metropolitanos, reemplazando las viejas creencias y las culturas locales, proporcionando un instrumento de control al poder central y debilitando el papel de la institución familiar, la comunidad, el grupo, la clase y el partido. Desde la anterior perspectiva, la diversidad se la considera como un valor amenazado por las tendencias que tienden a la centralización y universalización.

Hay otra versión alternativa sobre el papel que han tenido los medios de comunicación en relación al cambio social, que asocia a éstos de forma más positiva con la diversidad. Desde esta perspectiva, los medios de comunicación pueden ser considerados como favorecedores del cambio y del desarrollo de la libertad, frente al control, ya sea este de la comunidad local, la religión, o el poder político unificado. Los medios de comunicación han difundido innovaciones y formas culturales alternativas, han cambiado los poderes establecidos y han 
favorecido las novedades, las elecciones, la apertura y la fragmentación de los vínculos sociales opresivos. En las dos versiones anteriores, el valor de la diversidad está considerado de forma positiva.

Desde la teoría dominante del Estado democrático liberal, la diversidad es un componente básico, dado que en esencia, ésta es pluralista. Desde esta teoría política, las sociedades existen para promover los beneficios de sus miembros individuales, y lo que es bueno es variado y sólo se puede descubrir, a través de la libertad de expresión de los diferentes objetivos y soluciones a los problemas. La mayor cantidad de alternativas proporcionarán mejores prospectivas para el bienestar individual y colectivo.

El valor de la diversidad está fuertemente vinculado con el concepto del pluralismo. Este concepto tiene sus orígenes en el siglo XVIII y gradualmente se convirtió en un importante concepto político. Este concepto se convirtió como una referencia positiva de cualquier sistema político, era considerado como un concepto clave para contrarrestar el poder del Estado. Posteriormente, el pluralismo se ha identificado con los principios de la democracia liberal, en la cual diferentes grupos de intereses compiten por el poder y por el acceso a los recursos ${ }^{1}$

\section{Diversidad y Medios de comunicación}

Desde la perspectiva vinculado al concepto del pluralismo la libertad de los medios de comunicación, y la multiplicidad de opiniones, y el bien de la sociedad están intrínsecamente unidos. La teoría de la libertad de la prensa es esencialmente una teoría sobre la diversidad de los medios de comunicación.

El valor de la diversidad dentro de los medios de comunicación puede verse limitado por la acción centralizada de las fuerzas políticas, sociales y económicas. Se han esgrimido diferentes razones (desde ideologías diferentes) que intentan explicar la disminución de este valor en los medios de comunicación. Por un lado, el valor de la diversidad por la intervención del Estado, que tiene el poder de regular y controlar los medios de comunicación. Por otro, la diversidad puede decrecer si dentro de los medios de comunicación existe una tendencia a la concentración de la propiedad, esto tiende a crear un pequeño número de grandes medios de comunicación a gran escala, que se concentran en los mercados mediáticos más beneficiosos y proveen la mayoría de demandas, con consecuencias negativas para la multiplicidad de medios independientes y para la provisión de productos minoritarios. Por último, independientemente del control

1 Véase la evolución histórica del concepto de pluralismo en los trabajos de Dahl (1967) y Tumin y Plotch (1977) 
de la propiedad, se puede aminorar la diversidad cuando existen tendencias internas dentro de las organizaciones de los medios de comunicación que se han identificado con las dinámicas que tienden hacia la estandarización de la cultura y en favor del status quo político, que favorecen las repeticiones y los estereotipos, en la producción y difusión de las noticias y de la información.

Basándose en las anteriores razones, el valor de la diversidad se pretende promover dentro de los medios de comunicación, a través, de dos formas contradictorias. Una primera forma de aumentar el desarrollo de la diversidad se realiza mediante la ampliación de los canales existentes (ampliación de la oferta). Actualmente, en Europa occidental se ha intentado promover el valor de la diversidad con la creación de los sistemas mixtos de televisión. La segunda forma de acrecentar la diversidad se intenta, a través de la intervención pública, regulando la libertad de mercado.

Algunos autores ${ }^{2}$ consideran que la diversidad se puede desarrollar ampliamente dentro de los medios, si existe una total libertad dentro de éstos. Otros analistas $^{3}$ opinan, que el incremento de la diversidad se puede conseguir con menos libertad. Estas dos formas de entender la diversidad plantean, por un lado, una versión de la diversidad que considera que la libertad de mercado y las demandas del consumidor, serán las que desarrollarán el valor de la diversidad (la primera); y, por otro lado, la segunda versión especifica que los elementos y dimensiones de este valor necesitan una protección por parte de las legislaciones nacionales y de los poderes públicos.

Hasta el momento actual, las políticas comunicativas orientadas hacia los medios de comunicación, en los distintos países desarrollados han tendido a promover la diversidad de las opiniones, de las expresiones y de la cultura, mediante dos formas divergentes: la primera de éstas formas ha consistido en maximizar las oportunidades para ampliar la libertad en los medios de comunicación; y la segunda, se ha realizado, a través, de la intervención de los poderes públicos en el mercado.

El pluralismo en los medios de comunicación puede contribuir a desarrollar la diversidad de tres formas importantes: la primera forma reflejando las diferencias de la sociedad ${ }^{4}$; la segunda forma dando acceso a los diferentes puntos

2 Esta tesis la mantienen, por ejemplo, Fowler (1982) y Veljanoski (1989).

Véanse al respecto los trabajos de Costa Badia (1986); Blumler (1991, 1992); HOffmann-Riem (1987, 1992a, 1992b); The Broadcasting Reserach Unit (1989); entre otros.

4 Según P.Jacklin, el principio de «reflejar las diferencias de la sociedad», lo ha definido como «que existe diversidad de representación cuando la estructura de la diversidad en las comunicaciones corresponde con la estructura de la diversidad en la sociedad». Este autor pone énfasis en la diversidad de la realidad social la estructura de la diversidad en la sociedad». Este autor pone énfasis en la diversidad de la realidad social
que se refleja en el contenido de los medios de comunicación, aunque la estructura del sistema de los que se refleja en el contenido de los medios de comunicación, aunque la estructura del sistema de los
medios puede ser considerado en términos que reflejen las diferencias existentes en la estructura social medios puede ser conside. 
de vista; y por último, la tercera forma será ofreciendo un amplio tipo de elección de opciones en los medios de comunicación.

\section{Diversidad, televisión y servicio público}

El valor de la diversidad ha estado presente en todas las radios y televisiones públicas en Europa. Dentro de la filosofía de servicio público este valor estaba orientado hacia el acceso de los ciudadanos y de toda la sociedad a una información pluralista, más que a la libertad de los comunicadores. Se consideraba que la diversidad en el contenido de los programas y la accesibilidad de éstos para todos los segmentos de la audiencia debía ser establecida y salvaguardada.

La diversidad entendida como el pluralismo, tanto regional, lingüístico, político, cultural y en materia de gustos, fue uno de los valores que intentaron aplicar las televisiones públicas. Este valor desde la filosofía del servicio público consideraba que si la sociedad es diversa en su composición, cada uno de los sectores debe encontrar en la programación televisiva materiales que reflejen sus intereses y con los que se pueda identificar. Las televisiones de servicio público consideraban que la audiencia era heterogénea y difería en gustos e intereses, por lo tanto, debería disponer de una amplia gama de opciones. También, el pluralismo programático debería dar la posibilidad de que entraran en contacto las diferentes inquietudes y estilos de vida de otros individuos y de esta forma se fomentaría la comprensión y la tolerancia dentro de las sociedades democráticas.

En Gran Bretaña, la diversidad o el ideal de pluralismo se ha intentado llevar a la práctica promoviendo este valor no sólo en términos, de equilibrio político y representación regional, sino también, proporcionando diversidad de programas para diferentes gustos culturales, acceso a las minorías y promoviendo diferentes formas alternativas de financiamiento y control. Uno de los principales principios básicos de la televisión de servicio público en Gran Bretaña era el de «ofrecer programas de amplia diversidad a lo largo de un tiempo razonable y dirigidos a prácticamente todos los tipos de gustos, tanto grupos masivos como minoritarios» (The Broadcasting Research Unit, 1985: 3)

En la República Federal de Alemania, las diferentes leyes sobre radio y televisión incluyen el requerimiento que cualquier grupo que reivindique el acceso a un medio tiene que demostrar que éste representa a un colectivo político, religioso o filosófico que está presente en la sociedad.

La televisión en muchos países empezó como un sistema de servicio público, independiente del gobierno y financiado directamente por la audiencia. Sin embargo, los principios del servicio público no se han definido, ni ha existido una definición clara de lo que significaba el servicio público, hasta hace poco tiempo. El papel y la función de la televisión de servicio público se convirtió en un centro de discusión en distintos países durante la década de los setenta, ochenta 
y noventa. En Alemania, cuando en los años ochenta se planteó la introducción de la televisión comercial se examinó los posibles efectos de este tipo de televisión en el mercado, y el rol y la función de la televisión de servicio público. Se consideró que la televisión de servicio público tenía que tener el propósito de satisfacer las necesidades de la gente, y se consideró este tipo de televisión como un sistema crucial para seguir desarrollando la opinión pública democrática. Esto significa que la televisión de servicio público debía reflejar la diversidad existente de opiniones, y jugar un rol activo en crear esta opinión pública. Uno de los valores claves del concepto de servicio público en la televisión de Alemania es la diversidad, y se estableció en este país un sistema televisivo que asegurase un información y opinión plural (Kirche und Rundfunk (1986))

En las diferentes discusiones que se han producido en las dos últimas décadas sobre la definición sobre el servicio público en la televisión, se ha llegado a aceptar un dominio común para el servicio público de la televisión. El principio básico es que se debe servir al público, reconocer los derechos de los ciudadanos en la sociedad y actuar como la mejor fuente posible de información para apoyar el diálogo público. Los factores claves que las televisiones públicas deben desarrollar son: una completa independencia del poder político; basarse en el desarrollo de las diferentes dimensiones del valor de la diversidad; y por último, tender hacia la excelencia y la competencia profesional.

Para algunos académicos ${ }^{5}$, la protección de la televisión de servicio público debe estar vinculada con una filosofía en su programación que se base en la diversidad. Como las televisiones públicas tienden a concentrarse sobre unos tipos de programas para atraer a una gran audiencia, todos los servicios de televisión tenderán a proporcionar el mismo tipo de materiales, se estrechará el tipo de programación que la audiencia puede escoger. Blumler, Brynin y Nossiter han probado que las actividades de las televisiones están influenciadas por su sistema de financiación. Al analizar las políticas de programación de canales públicos y privados han llegado a la conclusión que los canales privados tienden a concentrar sus espacios sobre determinados géneros de programación. Esta conducta es lógica dado que ellos deben obtener beneficios para sobrevivir y ciertos tipos de programas apelan a una gran proporción de la audiencia más que otros.

La televisión pública no intenta buscar un mercado, de esta forma es capaz de proveer diversidad de programas para satisfacer las necesidades de la audiencia. En esta televisión, la diversidad es el valor clave, y puede ser un elemento persuasivo y de referencia positiva frente al público, sin embargo, el desarrollo de la era multicanal amenaza la lógica del servicio público.

5 Véase, Blumler, Brynin y Nossiter (1986). 


\section{Televisión comercial y diversidad}

Algunas de las responsabilidades que se han asignado a las televisiones de servicio público, también, se le han asignado a las televisiones comerciales. En una sociedad democrática, las televisiones comerciales deberían lograr un equilibrio entre obtener beneficios y los requerimientos de un servicio público. La extensión de este servicio público varía de un país a otro. En los Estados Unidos, este requerimiento está expresado por el Federal Communications (FCC), en su Blue Book, public service responsabilites of broadcasting licenses ${ }^{6}$. En Alemania hubo un amplio debate sobre este tema entre la década de los setenta y ochenta, y se estableció un sistema mixto, financiado por la publicidad, que debe servir al Grundversorgung $^{7}$, que debe estar al servicio del orden constitucional liberal y democrático. Este nuevo sistema dual de la televisión permitió a las televisiones comerciales un poco más de libertad en sus operaciones y ser capaces de sobrevivir mejor en el mercado. En Gran Bretaña, antes de la Ley de Televisión de 1990, las televisiones comerciales se les exigía que desarrollasen, las diversas responsabilidades asociadas con el servicio público bajo la supervisión de la autoridad reglamentadora, del IBA $^{8}$. Actualmente, el sistema ha cambiado, y las televisiones comerciales están bajo la supervisión del ITC ${ }^{9}$, y se les ha asignado unas obligaciones menos pesadas de servicio público. Originalmente, las televisiones comerciales estaban dentro de un sistema televisivo compuesto por pocos canales, donde el concepto de servicio público, implicaba, tanto a las televisiones comerciales como públicas. El sistema televisivo británico ha ido perdiendo el equilibrio y los valores que lo sustentaron durante un largo período, sobre todo al crearse una multiplicidad de canales que en la actualidad son disponibles a través de distintas vías.

\section{Tres perspectivas para estudiar las dimensiones de la diversidad}

El valor de la diversidad puede ser definido y estudiado a través de diferentes perspectivas de análisis, donde se analizan las distintas dimensiones que tiene este concepto: la primera perspectiva de investigación la han creado los analistas Denis Mcquail y Van Cuilenburg; la segunda y la tercera perspectiva las ha elaborado, el académico alemán Wolfgang Hoffmann-Riem.

Denis Mcquail y Van Cuilenburg han identificado seis dimensiones del valor de la diversidad, que deben ser tenidas en cuenta cuando se examina este valor. Estas dimensiones son las siguientes: la primera dimensión de la diversidad,

6 Federal Communication Commission (1946)

Grundeversorgung significa literalmente "servicio de base".

IBA (Independent Broadcasting Authorty): Autoridad Independiente de la Radio y la Televisión. Esta institución británica desapareció en 1990, con la instauración de la Ley de Televisión de !990.

ITC (Independent Television Commission): Comisión Independiente de la Televisión. Esta institución se creo mediante la Ley de Televisión de 1990 
diversidad en las televisiones públicas y comerciales

está en relación a las diferentes áreas sociales de una sociedad: el área política, socioeconómica y sociocultural; según estos autores, la segunda dimensión se estudiará de acuerdo con la dimensión de pluralidad social escogida: en términos de las diferencias que puedan ser descritas entre los distintos grupos de una sociedad; la tercera dimensión de la diversidad se estudiará, a partir de las funciones de los medios de comunicación: información, formación de opiniones, etc. Esta dimensión proporciona un puente conceptual entre la diversidad de los medios de comunicación y la diversidad social.

$\mathrm{Si}$ se realiza un examen de la literatura sobre las funciones principales que cumplen los medios de comunicación, éstas pueden resumirse en seis tareas que los medios cumplen para los diferentes grupos sociales: la primera función es la de proporcionar información; la segunda función es la de ofrecer actividades (programas) para la formación de opiniones, que ayudan a la interpretación de la experiencia y la elaboración de juicios de valor; la tercera función es la de expresión, que se puede definir como "la provisión de una plataforma de canales para que los individuos o grupos puedan expresar sus puntos de vista y desarrollar su propia identidad cultural y social"; la cuarta función es de crítica -esta función se reconoce más claramente dentro de la esfera política, pero también está presente en las esferas socio-económicas y socioculturales, cuando emergen diferentes ideas y creencias en conflicto-; la quinta función es la del entretenimiento -es importante reconocer que una gran cantidad del contenido de los medios de comunicación consiste en entretenimiento y diversión-; la sexta función es la de movilización, que se define como "el uso de los medios de comunicación para dinamizar o activar a las personas sobre determinados problemas o objetivos". Esta función es más reconocible en la esfera política, pero también puede encontrarse en la vida cultural, social y económica (Mcquail y Van Cilenburg, 1983: 153-154)

La cuarta dimensión de la diversidad examina los diferentes niveles del sistema de los medios de comunicación. El sistema de medios de comunicación se puede estudiar en relación a tres niveles diferentes. El primer nivel es el micro, es decir, el estudio de las características de un sólo medio de comunicación. El segundo nivel es el meso, en éste se puede estudiar las características de un tipo de medio de comunicación (Ej: la televisión). El tercer nivel es el macro, en éste se estudia el sistema de medios de comunicación en su conjunto (Mcquail y Cuilenburg, 1983: 151). La quinta dimensión está en relación a los diferentes contendidos de los medios y a las dimensiones de una sociedad plural; y, por último la sexta dimensión se puede estudiar a partir de una pluralidad de criterios. Esto quiere decir que se puede medir el valor de la diversidad, a partir de lo que determinemos y ver si los medios de comunicación desarrollan este criterio, de una forma plural (Mcquail y VanCuilenburg, 1983: 147-154)

Wolfgang Hoffmann-Riem ha elaborado dos clasificaciones sobre las dimensiones del valor de la diversidad. En la primera clasificación, este autor 
identifica cuatro dimensiones del valor de la diversidad. La primera dimensión está en relación a los formatos y metas -esencialmente se refiere a las diferentes funciones que deben cumplir los medios de comunicación, como el de proporcionar información, educación y entretenimiento; la segunda dimensión examina los contenidos -los tópicos de información, opinión y noticias-; la tercera dimensión estudia la diversidad, vinculándo ésta con el acceso y la representación de grupos y personas en los medios de comunicación; la cuarta dimensión, está en relación con el espacio geográfico que se cubre y a la importancia que se le da a éste (Hoffmann-Riem, 1987:61)

En la segunda clasificación, Hoffmann-Riem considera que el valor de la diversidad comprende como mínimo cinco dimensiones ${ }^{17}$ : la primera dimensión está en relación a la expresión de una pluralidad de opiniones. Esta diversidad examina los programas orientados a la opinión, es decir, en particular, a la cobertura de opiniones sobre temas de relevancia social, y la exclusión de un poder que influya unilateralmente sobre la formación de opiniones; la segunda está en relación a la presentación de posturas y perspectivas de colectivos sociales diversos. Es una diversidad orientada al individuo y al grupo: es decir, es aquella que ofrece a las fuerzas y grupos sociales de importancia, la oportunidad de expresarse; la tercera dimensión estudia la cobertura de temas de interés general. Es una diversidad orientada a los temas, es decir, aquella que cubre con programas una gama de tópicos suficientemente amplia; la cuarta dimensión se vincula con el reflejo de los intereses territoriales. Es la diversidad que refleja la cobertura de opiniones y noticias de áreas locales, regionales, nacionales y supranacionales; y por último la quinta dimensión estudia la incorporación de una diversidad de formatos. A través de esta diversidad podemos examinar la provisión equilibrada de los diversos géneros de programas, en particular, información, entretenimiento, educación y consejos (Hoffmann-Riem, 1992a:74).

6. La diversidad como un elemento de la calidad: otras reflexiones e investigaciones para estudiar la diversidad

La diversidad ha sido uno de los conceptos claves vinculados a la televisión de servicio público. Una de las funciones claves de la institución pública de televisión ${ }^{10}$ es reflejar la diversidad existente de opiniones, ofreciendo una fuente de información plural y diversa, y una programación general basada en el concepto de diversidad, que permita satisfacer las necesidades de los telespectadores.

10 En concepto de diversidad ha tenido una enorme relevancia en las televisiones públicas de Alemania y Japón. En estas dos instituciones públicas, la noción de diversidad ha sido la idea clave de la televisión de servicio público. 
La mayoría de las reflexiones e investigaciones que se han realizado sobre la calidad coinciden en afirmar que el sistema televisivo debe ofrecer una amplia tipología de programas, que deben diversificarse como géneros, como contenido, como tipologías, estilos, como posiciones y opiniones a expresar. En distintas investigaciones, se ha estudiado la diversidad como un elemento de calidad en la programación televisiva y se han elaborado distintos métodos e indicadores para evaluar la diversidad.

La diversidad como un elemento de calidad tiene la ventaja de que no es tan subjetivo, y es posible medir con una cierta precisión la diversificación de una programación de una red vertical o de un sistema horizontal, a través de una serie de indicadores empíricos: la hora dedicada a un tipo de programa; los recursos económicos y profesionales destinados a diferentes tipos de programas; el tiempo y el espacio dedicado a un grupo social, étnico; entre otros indicadores.

En Suecia un criterio general de la calidad es el de diversidad. Diversidad implica que los resultados del programa total deben tener una serie de dimensiones: tema, genero, perspectiva, grupo de referencia (definido en función de características demográficas, socioeconómicas, étnicas o geográficas, pero también, en función de gustos, intereses, necesidades, predisposiciones, etc.). Esta diversidad no se refiere simplemente a la variedad o multiplicidad de un nivel general, sino que está directamente relacionado con el reconocimiento de la complejidad de la realidad social y a la consideración de los intereses minoritarios en un sentido amplio. En este país, el criterio de diversidad se vincula directamente con la programación doméstica. Desde la perspectiva sueca, un servicio de alta calidad debe incluir una proporción alta de producción doméstica. En este país, el sistema de televisión se ha diseñado para promover la calidad de acuerdo a diferentes criterios. El primer criterio es el de diversidad en la representatividad social. A pesar de las tendencias a la homogeneización inducidas por la competencia por las audiencias y por la internacionalización de la cultura de los medios de comunicación, la televisión ofrece una gran variedad de mensajes y programas. Gran cantidad de dinero se ha dedicado a ofrecer un cierto tipo de diversidad. Los más claros beneficios del sistema televisivo se pueden apreciar en los servicios informativos que se ofrecen: noticias, actualidad, documentales (40\% del total de la programación $)^{11}$.

La diversidad de la oferta televisiva se puede analizar a través de siete dimensiones (Blumler 1986, 1991b, 1992). La primera dimensión es la diversidad sustancial y se compone de diversos aspectos. El primero está vinculado con la diversidad de opiniones, de tendencias y de puntos de vista más importantes de una sociedad que se pueden encontrar en la programación televisiva. El segundo

11 Véase al respecto el trabajo de Nowak (1991: 253). 
aspecto se refiere al desarrollo de una programación que permita a los diversos grupos (sociales, económicos, por sexo, etc.) de ser visibles y de expresarse en la televisión. El tercer aspecto hace referencia a la diversidad en relación a los argumentos y los temas tratados. Con el último aspecto se vincula el abanico de valores que ofrece un programa o una red de televisión.

La segunda dimensión es la diversidad del tipo de programa. Esta dimensión se define como el grado de diversificaciones de géneros televisivos que componen una programación (noticias, ficción, información, etc.). Se trata de la tentativa de no imitar los programas de genero amplio, de evitar las repeticiones y la estandarización de formatos. La tercera dimensión es la diversidad del palimpsesto. En relación a este tipo de diversidad se pueden distinguir dos dimensiones: a) una diversidad interna en una red (diversidad vertical); y b) otra que hace referencia al sistema televisivo y su complejidad (diversidad horizontal). En el primer caso, la diversidad se calcula midiendo cómo los distintos géneros de programas se han distribuido en un palimpsesto durante un día, u otro tiempo. En el segundo caso, la diversidad se mide teniendo en cuenta la amplitud de varias redes de televisión, en una determinada franja horaria.

La cuarta dimensión es la diversidad estilística. Esta dimensión se puede definir como un indicador que revela dónde y cuántos programas se han encontrado las condiciones y el espacio para desarrollar según un estilo específico, con sus características y valores. Esta diversidad está en oposición con la tendencia a llenar la programación de modelos de programas estandarizados, repitiendo estilos, formas expresivas o consolidando, las que en un momento dado están de moda, o han obtenido los mayores índices de audiencia. La quinta dimensión es la diversidad de la distribución de recursos. Según este tipo de diversidad, los diferentes tipos de programas deben tener un nivel de recursos, en términos monetarios, de medios técnicos, artísticos, y de distribución dentro de una red de televisión.

La sexta dimensión es la diversidad de la audiencia. Esta dimensión se compone de diversos aspectos que responden a demandas diversas. En una programación televisiva hay muchos programas que se conciben y realizan para un segmento de público delimitado. En general, en la transmisión televisiva se pueden distinguir dos grandes categorías: a) una está constituida de los programas que para ser seguidos no se demanda ningún esfuerzo por el espectador; y b) otra que demanda al telespectador una participación muy atenta. La séptima y última dimensión es la diversidad de la calidad. Este aspecto de la diversidad presupone el hecho de que, aunque la noción de calidad se articule en muchas dimensiones, ésta puede ser definida como "característica de la calidad". Esto conlleva que quien produce la televisión puede trabajar para lograr diversos tipos de "resultados óptimos o de excelencia", a partir de los cuales su trabajo puede ser valorado. Desde este punto de vista se puede considerar que un sistema de televisión debe 
tener presente junto a la amplitud y a la diversificación, el estímulo de la calidad. Desde esta perspectiva la valoración de un sistema televisivo no se considera únicamente en base al nivel de su programación sino en relación a cuántos estándares de calidad promueve.

Sobre los métodos y los indicadores para medir la diversidad, se han elaborado aportaciones interesantes, a través de las investigaciones que se han hecho, en Estados Unidos sobre el tema ${ }^{12}$. También, se ha realizado una importante reflexión sobre el tema de la diversidad como producto económico, en particular sobre la relación entre la demanda y la oferta ${ }^{13}$. En Europa, la investigación sobre la oferta televisiva y la calidad (diversidad) se ha retardado por las condiciones de los monopolios televisivos ${ }^{14}$.

\section{Diversidad, Era Multicanal, Internet y televisión pública}

Actualmente, existe un sistema televisivo formado por múltiples canales, donde el público puede escoger el que más le satisface. Los canales operan comercialmente y la libre competencia determina la mejor combinación del servicio para la audiencia. Este modelo se ha impuesto, hoy en día, y es apoyado por intereses que benefician a éste.

El sistema multicanal, en sí mismo tiene algunas deficiencias que vulneran la diversidad dentro de este sistema. Algunos estudios recientes ${ }^{15}$, han probado que un número grande de canales no asegura la diversidad dentro del sistema de televisión. Un incremento en el número de canales no proporciona una diversidad del servicio. Sonnerberg considera que "la afirmación de que la competencia proporciona diversidad se ha mantenido, haciendo que la diversidad sea un producto de la competencia por definición(...). Contrariamente a los que apoyan la teoría del mercado de la televisión, los resultados de los estudios de economía de la televisión demuestran que el incremento del número de canales con un base de financiación diferente, puede ser capaz de proporcionar diversidad sólo en un sentido muy limitado del término" (Sonnenberg 1993:87).

Algunos operadores argumentan que la propiedad de muchos canales permite proporcionar una programación diversa, y que ellos pueden operar y aceptar una pérdida en un canal compensándolo con otro más rentable. Sonnerberg considera que diversidad de servicio no significa diversidad cultural, y además, aunque el operador pueda mantener un canal que no le proporciona beneficios,

12 Sobre los métodos de medición del valor de la diversidad destacamos los trabajos de Dominick y Pearce (1976); Litman (1979); Owen (1977) y Adams y Wakshlag (1985)

13 Sobre este tema destacamos los trabajos de Litman (1979 y 1992).

14 Los principales estudios que se han realizado sobre la calidad (diversidad) en Europa se han hecho en el Reino Unido. Los trabajos más relevantes sobre este tema son los siguientes, Wober $(1988,1989,1990)$ Wober y Kilpatrik (1988).

15 Véase, Sonnenberg (1993) 
mientras él continua teniendo beneficios en otros canales, si la situación financiera empeorase, no existe duda que los canales que no proporcionasen beneficios serían abandonados antes que los otros. La diversidad proporcionada por un número de canales de un mismo propietario es una ilusión.

En un sistema multicanal, se podría proporcionar diversidad, aunque ésta no sería un servicio adecuado para la audiencia, debido a que la gente tiene una limitada capacidad de procesar información. Los estudios realizados en Estados Unidos sobre la audiencia indican que los telespectadores no pueden usar muchos canales, y un porcentaje de individuos puede mirar menos de diez ${ }^{16}$.

Cuando el número de canales excede un número, el público tiende a dar forma a su propio repertorio, su "repertorio de canales", para satisfacer sus necesidades. El tipo de servicios incluidos en este repertorio de canales difiere de un individuo a otro. La importancia de este punto aquí es que hay un limitado número de canales que un individuo puede reconocer y usar. Si el repertorio de canales contiene menos de diez canales y todos los canales son especializados, el público sólo consumirá un número restringido de variedad de programas.

El tema del "repertorio de canales", también, se ha estudiado en Japón Ishikawa 1996:101). En los estudios realizados en este país se ha confirmado nuestra limitada capacidad para usar canales múltiples, dentro de un sistema televisivo multicanal. El esfuerzo y el tiempo que podemos dedicar a recibir información que necesitamos diariamente es limitado. Si toda el tipo de información que necesitamos está extendida en cien canales, la búsqueda de esta información necesitaría un tiempo y un esfuerzo que excede nuestra capacidad y habilidad.

Las guías de programación de la televisión no son una fuente de información útil para el público, dado que podemos mirar lo que programan en los canales más importantes, pero no podemos mirar lo que programan en cien canales. Por lo tanto, debido a esta situación, el público se sienta frente a la televisión y busca algo para mirar con el mando a distancia. Si todos los canales son especializados, será imposible proporcionar una completa guía de televisión para el público, dado que éste se verá saturado por el número de canales y perderá la capacidad de escoger de forma efectiva. Un sistema televisivo multicanal puede tener diferentes tipos de información, pero para el público puede resultar difícil encontrar la información que busca y necesita. Se demuestra que es poco realista esperar que una gran cantidad de canales especializados proporcionen un sistema televisivo con diversidad. Los canales no pueden ser usados por completo. Los estudios realizados en Japón consideran que el modelo multicanal especializado no funcionará.

16 Véase, Heeter (1988) 
diversidad en las televisiones públicas y comerciales

La televisión de servicio público que se financia de forma directa por los telespectadores y proporciona una diversidad de información tiene una razón de existir en la era multicanal, donde las nuevas tecnologías, exigen un nuevo papel para las instituciones públicas de televisión. Es importante tener en cuenta en esta nueva era, que la creación y el desarrollo de la tecnología innovadora de la red Internet está produciendo una estructura totalmente diferente de comunicación, dentro del sistema de medios global.

Hasta hace poco, el acceso a la tecnología que permite a las personas comunicar un mensaje a una gran audiencia estaba restringida a un número pequeño de gente porque los medios eran muy costosos para tener y operar. Por estas razones, existen regulaciones y requerimientos creados sobre los que poseen un medio capaz de llegar a una larga audiencia. El sistema de medios está organizado en esta dirección, y podemos esperar un cierto grado de competencia ética y profesional en la creación de mensajes comunicados a través de los medios de comunicación.

La estructura de la red Internet es completamente diferente a las estructuras de los medios de comunicación anteriormente existentes. En esta nueva red, cada individuo puede ahora difundir un mensaje a una larga audiencia a través de una red. Por un lado, esta capacidad puede ser un paso importante hacia un sistema democrático más avanzado donde cada individuo puede enviar un mensaje a un número enorme de gente. Por otro lado, esta capacidad puede amenazar seriamente la manera de obtener información. Como no existe ninguna regla que regule esta actividad de los individuos, un mensaje difundido por Internet puede llegar a cualquier lugar. Este puede ser un rumor, libelo o una infracción de la privacidad. Teniendo en cuenta la cantidad y rapidez de los flujos informativos, los medios encontrarán difícil satisfacer esta función de confirmación. La prensa, por ejemplo, puede que no sea capaz de proveer de forma rápida la confirmación para sus lectores. La televisión, en el otro lado, puede recibir peticiones de los telespectadores que hubieran perdido un mensaje al emitirse. Todos los medios que existen pueden ser requeridos para empezar un nuevo servicio que llene este papel de confirmación. Un método práctico puede ser abrir un página web en Internet y proveer de la información que se necesita revisar. La páginas webs usada por la gente como fuentes de confirmación, las más importantes serán las páginas webs proporcionadas por las televisiones de servicio público.

En esta nueva era multicanal, la televisión de servicio público será una de las fuentes mejor cualificadas para confirmar la información que surge en Internet, porque es independiente y tiene las capacidades profesionales necesarias. La televisión de servicio público, por definición, debe servir al público. Ésta necesita tomar la iniciativa y llenar este nuevo rol de fuente confirmativa, de calidad y donde la diversidad sean sus valores añadidos y diferenciadores. Si esto sucede en este campo, será otra razón para estar en este nuevo panorama de nuevos medios. 
Conclusiones

La diversidad como valor positivo dentro de los medios de comunicación ha sido comprendida desde diferentes perspectivas. Algunos analistas consideran que la diversidad se puede desarrollar dentro de los medios a través de políticas comunicativas que tiendan a crear una gran libertad de actuación en el sistema mediático. Desde esta posición la diversidad está asociada con la libertad de mercado y a las demandas del consumidor. Otros autores plantean que las dimensiones de este valor se promueven, mediante intervenciones de los poderes públicos y la regulación del mercado. Desde este planteamiento se considera que las distintas dimensiones de la diversidad necesitan una protección por parte de las legislaciones nacionales y de los poderes públicos.

Las políticas comunicativas que se han desarrollado en los distintos países desarrollados, orientadas hacia los medios de comunicación, han tendido a promover la diversidad de las opiniones, de las expresiones y de la cultura, mediante dos formas divergentes: la primera de éstas formas ha consistido en maximizar las oportunidades para ampliar la libertad en los medios de comunicación; y la segunda, a través de la intervención de los poderes públicos en el mercado.

Dentro de los medios de comunicación se puede desarrollar la diversidad de tres formas importantes: la primera forma reflejando las diferencias de la sociedad, la segunda forma dando acceso a los diferentes puntos de vista; y por último, la tercera forma será ofreciendo un amplio tipo de elección de opciones en los medios de comunicación.

En la mayoría de las reflexiones e investigaciones que se han realizado sobre la diversidad se considera que el sistema televisivo debe ofrecer una amplia tipología de programas, que deben diversificarse como géneros, como contenido, como tipologías, estilos, como posiciones y opiniones a expresar. En distintas investigaciones, se ha estudiado la diversidad como un elemento de calidad en la programación televisiva y se han elaborado distintos métodos e indicadores para evaluar la diversidad.

El estudio de la diversidad tiene la ventaja de que es un valor más objetivo, dado que es posible medir con un cierta precisión la diversificación de una programación de una red vertical o de un sistema horizontal, a través de una serie de indicadores empíricos: la hora dedicada a un tipo de programa; los recursos económicos y profesionales destinados a diferentes tipos de programas; el tiempo y el espacio dedicado a un grupo social, étnico; entre otros indicadores.

La diversidad ha sido uno de los conceptos claves vinculados a la televisión de servicio público. Una de las funciones claves de la institución pública de televisión es reflejar la diversidad existente de opiniones, ofreciendo una fuente de información plural y diversa, y una programación general basada en el concepto de diversidad, que permita satisfacer las necesidades de los telespectadores. 
La televisión de servicio público que se financia de forma directa por los telespectadores y proporciona una diversidad de información tiene una razón de existir en la era multicanal, donde las nuevas tecnologías, exigen un nuevo rol para las instituciones públicas de televisión. Es importante tener en cuenta en esta nueva era, que la creación y el desarrollo de la tecnología innovadora de la red Internet está produciendo una estructura totalmente diferente de comunicación, dentro del sistema de medios global.

En la nueva era multicanal, la televisión de servicio público será una de las fuentes mejor cualificadas para confirmar la información que surge, en Internet y en otros medios de comunicación, si logra convertirse en la mejor fuente posible de información y contribuye a desarrollar el diálogo público. En este contexto, los valores básicos que deberán impulsar las instituciones públicas de televisión serán: la independencia política, la diversidad, y la excelencia y competencia profesional. La televisión de servicio público, por definición, debe servir al público, y tomar la iniciativa para llenar este nuevo rol de fuente contrastada y veraz, donde la calidad y la diversidad sean los valores añadidos y diferenciadores.

\section{Bibliografía:}

- ADAMS W.J y WAKSHLAG, J, (1985): "Trends in Program Variety and the Prime Time Access Rule», Journal of Broadcasting, Vol.29, nº1, pp. 23-34.

- BLUMLER, Jay. G, (1989):The Rol of Public Policy in the New Television Marketplace,

- BENTON, Fondation, Washington D.C.

(1990): «Elections, the Media and the Modern Publicity Process», en FERGUSON, MARJORIE (ed), Public Com-munication: the New Imperatives, Sage, London/Newbury Park/New Delhi, pp. 101-113.

(1991a): «Television in the United States: Funding Sources and Programming Consequences» en, BLUMLER, JAY.G y NOSSITER, Thomas Johnson (eds), Broadcasting Finance in Transition. A Comparative Handbook, Oxford University Press, Oxford/New York, pp. 41-94.

(1991b): «In Pursuit of Programme Range and Quality», Studies on Broadcasting, Theoretical Research Center, NHK Broadcasting Culture Research Institute, Tokio.

(1992a): «Introducción: Confrontaciones actuales en la televisión de Europa Occidental», en BLUMLER, Jay.G (ed), Televisión e Interés Público, Bosch, Barcelona, pp. 11-17.

(1992b): «La televisión pública ante el diluvio comercial», en BLUMLER, Jay.G, Televisión e Interés público, Bosch, Barcelona, pp. 21-38.

(1992c): «Los valores vulnerables en juego», en BLUMLER, Jay. G (ed), Televisión e Interés público, Bosch, Barcelona, pp. 39-63. 
- BLUMLER, Jay. G, BRIYNIN, MALCOLM y NOSSITER, Thomas Johnson, 1986, «Broadcasting Finance and Programme Quality: an International Review», European Journal of Communication, Vol.1, No3, pp. 343-364, Routledge, London.

- BROADCASTING RESEARCH UNIT, 1985, The Public Service Idea in British Broadcasting: Main Principles, Broadcasting Research Unit, London. (1989): Quality in Television: Programmes, Programmemakers, Systems, John Libbey, London/París.

- COSTA BADIA, Pere Oriol, (1986): La crisis de la televisión pública, Paidós, Barcelona.

- DAHL, R.A, (1967: Pluralist Democracy in the US: Conflict and Consent, Rand McNally, Chicago.

- DOMINICK, J.R y PEARCE, M.C, (1976): «Trends in Network Primetime Programming», Journal of Communication, Winter, vol.26, № 1 .

- FEDERAL COMMUNICATION COMMISSION, 1946, Blue Public, Service Responsabilities of Broadcasting Licenses, FCC, Washington DC.

- HOFFMANN-RIEM, Wolfgang, (1981a): Kommerzielles Fersehen: Rundfunkfreiheit zwischen okonomischer Nutzung und staatlicher Regelungsverantwortung: das Beispiel, Nomos, Baden-Baden.

(1981b): «The Freedom of Communication and the Future of Broadcasting in West Germany», Studies of Broadcasting, Nº17, pp.49-70.

(1987): «National Identity and Cultural Values: Broadcasting Safeguards», Journal of Broadcasting, $\mathrm{N}^{\circ} 31$, pp. 57-72.

(1992a): «La protección de valores vulnerables en el ordenamiento televisivo alemán, en BLUMLER, Jay. G, Televisión e Interés público, Bosch, Barcelona, pp. 67-87.

(1992b): La defensa de valores vulnerables: medidas reguladoras y problemas de aplicación», en BLUMLER, Jay.G, Televisión e Interés Público, pp. 223256.

- HEETER, Carrie, (1988): "The Choice Process Model", en HEETER, C y GREENBERG (eds), Cable Viewing, Ablex, Norwood, Ablex.

- ISHIKAWA, Sakae, (1996): «The rol and function of public service broadcasting in a multichannel media environment», The Public/Javnost, Journal of the European Institute for Communication and Culture, vol. III, N ${ }^{\circ}$ 2, pp. 97-107, Ljubljana (Eslovenia), University Luton Press.

- Jacklin, P, (1978): "Representative Diversity", Journal of Communication, No28, pp.85-88.

- KIRCHE UND RUNDFUNK, (1986): No 88. Litman, Barry. R, 1979,»The Television Networks, Competition and Program Diversity», Journal of Broadcasting, vol. 23, Primavera, pp. 393-409. 
(1992): «Economics Aspects of Program Quality: the Case for Diversity», Studies on Broadcasting, Theoretical Research Center, NHK Broadcasting Culture Research Institute, Tokio.

MCQUAIL, Denis, (1991): "Broadcasting Structure and Finance: the Netherland", en BLUMLER, Jay.G y NOSSITER, Thomas. J (eds), Broadcasting Finance in Transition. A Comparative Handbook, Oxford University Press, Oxford/New York, pp. 144-157.

(1992): Media performance: mass communication and the public interest, Sage, London.

(1992b): «Los Países Bajos: libertad y diversidad bajo condiciones de variedad de canales», en Blumler, Jay. G (ed), Televisión e Interés Público, Bosch, Barcelona, pp. 131148.

- Mcquail, Denis y VAN CUILENBURG, Jan.J, (1983): «Diversity as a Media Policy Goal: a Strategy for Evaluative Research and a Netherlans Case Study», Gazette, № 31, pp. 145162.

- NOWAK, Kjell, (1991): "Television in Sweden 1986: Position and Prospects", en Broadcasting Finance in Transition: a Comparative Handbook, Oxford University Press, Oxford/New York, pp. 235-259.

OWEN, B.M, (1977): «Regulating Diversity: the Case of Radio Format», Journal of Broadcasting, Verano, Vol.21, No 3, pp. 305-319.

- SONNENBERG, Urte, (1993): "Channel Multiplicity and Programme Diversity in Television: Two Sides of tge Same Coin?", Stuidies of Broadcasting $\mathrm{n}^{\circ}$ 29, pp. 71-92.

- TUMIN, M.M, y PLOTCH, W (eds), (1977): Pluralism in a Democratic Society, Praeger, New York.

- Veljanovski, Cveto (de), (1989): Freedom in Broadcasting, Institute of Economics Affairs, London.

- WOBER, J.M, (1988): The Use and Abuse of Television, Lawrence Erlbaum Associated, Hillsdale, N.J.

(1989): «The UK: the Constancy of audience Behavior», en BECKER, I.B, et al (eds), Audience Response to Media Diversification, Lawrence Erlbaum Associated, Hillsdale, N.J. 1990,The Assesment of Television, IBA, Research Paper, Julio, London.

- WOBER, J.M y KILPATRIC, E, 1988, The Cost of Choice: a Calculus of Programme Want, Variety and Waste, IBA, Paper, Marzo, London. 